\title{
Estructura y regeneración de bosques de Prumnopitys andina en los Andes del sur de Chile
}

\author{
Structure and regeneration of Prumnopitis andina forests in the Andes of southern Chile
}

\section{Rodrigo Vargas-Gaete ${ }^{1 *}$, Christian Salas-Eljatib ${ }^{2}$, Diego Penneckamp ${ }^{3}$, Zoia Neira ${ }^{1}, M$. Cristina Diez ${ }^{4} \&$ Ricardo Vargas-Picón ${ }^{5}$}

${ }^{1}$ Departamento de Ciencias Forestales, Universidad de La Frontera, Temuco, Chile.

${ }^{2}$ Centro de Modelación y Monitoreo de Ecosistemas, Universidad Mayor, Santiago, Chile.

${ }^{3}$ Laboratorio de Biodiversidad y Ecología del Dosel, Facultad de Ciencias Forestales y Recursos Naturales, Universidad Austral de Chile, Valdivia, Chile.

${ }^{4}$ Departamento de Ingeniería Química, Universidad de La Frontera, Temuco, Chile.

${ }^{5}$ Consultor independiente, Chile.

*E-mail: rodrigo.vargas@ufrontera.cl

\section{RESUMEN}

Prumnopitys andina (lleuque) es una especie de conífera con una distribución restringida en Chile y Argentina. A pesar de su importancia ecológica, los conocimientos sobre la estructura y dinámica de los bosques donde crece $P$. andina son limitados. El objetivo de este trabajo fue describir la estructura y regeneración de bosques con alta presencia de P. andina. Se muestrearon bosques cercanos a Lonquimay (38¹' S, 71 ${ }^{\circ} 3^{\prime} \mathrm{O}-38^{\circ} 3^{\prime} \mathrm{S}, 71^{\circ} 3^{\prime}$ O) midiendo árboles, regeneración y flora vascular a través de 24 parcelas. Se encontró que $P$. andina ocurre predominantemente en el estrato superior (15-18 m) acompañado por Nothofagus obliqua y/o Austrocedrus chilensis, además de Lomatia hirsuta y Maytenus boaria en estratos intermedios (4-8 m). En base a la estructura, se identificaron tres tipos de bosques de $P$. andina: bosques abiertos, bosques secundarios adultos y bosques puros. Los bosques puros de $P$. andina o "lleuquerías" alcanzaron áreas basales entre las más grandes reportadas para bosques templados del sur de Sudamérica $\left(230 \mathrm{~m}^{2} / \mathrm{ha}\right)$. La regeneración de $P$. andina osciló entre 1.550 individuos/ha en bosques abiertos y 25.000 individuos/ha en bosques puros. $P$. andina exhibió una estrategia de regeneración aparentemente continua, y en ausencia de disturbios antrópicos como pastoreo de ganado o tala selectiva, sus poblaciones serían autosuficientes.

Palabras clave: arilo carnoso, lleuque, Lonquimay, Podocarpaceae.

\section{ABSTRACT}

Prumnopitys andina is a conifer tree species with a narrow distribution in Chile and Argentina. Despite its ecological importance, knowledge about the structure and dynamics of the forests where $P$. andina grows is limited. We aim to describe the structure and regeneration of forest with high presence of $P$. andina. Forests near Lonquimay (38 $1^{\prime} \mathrm{S}, 71^{\circ} 3^{\prime} \mathrm{W}-3^{\circ} 3^{\prime} \mathrm{S}, 71^{\circ} 3^{\prime} \mathrm{W}$ ) were sampled by measuring trees, regeneration and vascular flora through 24 plots. We found that $P$. andina predominantly occurs in the upper canopy (15-18 $\mathrm{m})$, usually in company of Nothofagus obliqua and/or Austrocedrus chilensis, in addition to Maytenus boaria and Lomatia hirsuta that occur in the mid-canopy (4-8 m). Based on forest structure, we identified three types of $P$. andina forests: open, secondary-mature, and pure. Pure $P$. andina forests reached basal areas among the largest reported for for temperate forests southern South America ( $230 \mathrm{~m}^{2} / \mathrm{ha}$ ). P. andina regeneration ranged from 1,550 individuals/ha 
in open forests to 25,000 individuals/ha in pure forests. P. andina exhibited a continuous regeneration strategy, which in the absence of human disturbances, such as grazing by cattle or selective logging, suggests that its populations are self-sustaining.

Keywords: fleshy cone, lleuque, Lonquimay, Podocarpaceae.

\section{INTRODUCCIÓN}

Prumnopitys andina (Poepp. ex Endl.) de Laub., lleuque, es una conífera de la familia Podocarpaceae, nativa de los bosques del centro-sur de Chile y de los Andes adyacentes de Argentina (Rodríguez 2004). Según lo reportado por Hechenleitner et al. (2012) y Rodríguez et al. (2005), puede alcanzar unos $15 \mathrm{~m}$ de altura y diámetros de hasta $100 \mathrm{~cm}$. Es una especie usualmente dioica, con inflorescencias masculinas y femeninas dispuestas en amentos que aparecen en noviembre, madurando sus semillas entre enero y marzo (Rodríguez et al. 1983). Las semillas se encuentran en conos drupáceos de forma ovalada de 1 a $2 \mathrm{~cm}$ de diámetro, envueltas por un arilo carnoso blando y pulposo, que es comestible y que se asemeja a una uva en su madurez, con el cual se preparan mermeladas y conservas (Rodríguez 2004, Hechenleitner et al. 2012). Este arilo carnoso denominado "uva de la cordillera" es comercializado localmente en mercados y ferias de manera predominantemente informal (Hechenleitner et al. 2012).

Lleuque se encuentra principalmente en la precordillera de Los Andes, distribuyéndose desde la provincia de Linares $\left(35^{\circ} 52^{\prime} \mathrm{S}\right)$ hasta la provincia de Cautín (39³0'S) entre los 200 y 1.380 m s.n.m (Rodríguez 2004, Hechenleitner et al. 2012). Presenta también una pequeña población disyunta remanente en la Cordillera de la Costa, en Nahuelbuta (Amigo et al. 2010, Hechenleitner et al. 2012). A lo largo de su distribución, lleuque participa en los tipos forestales: Ciprés de la Cordillera, RobleHualo, Roble-Raulí-Coihue y Coigüe-Raulí-Tepa (Donoso 1981, Rodríguez 2004, Hechenleitner et al. 2012). En base a la clasificación vegetacional de Luebert \& Pliscoff (2017) se encuentra asociado a varios "pisos de vegetación", ocurriendo de forma predominante en la asociación Nothofago obliquaePrumnopitydetum andinae (Amigo et al. 2010), la cual se inserta como una comunidad zonal en varios pisos, principalmente asociada a bosques caducifolios templado andinos de Nothofagus alpina-N. obliqua, Nothofagus alpina-N. dombeyi; N.pumilio-Araucaria araucana; N.pumilio-Azara alpina; así como también, asociada al bosque resinoso templado andino de $A$. araucana-N. dombeyi (Luebert \& Pliscoff 2017).

La mayor distribución de la especie se encuentra en la región de La Araucanía (49,6 \%), seguido por la región del
Biobío (48,2 \%), abarcando en total unas 9.300 ha (CONAF 2015). En La Araucanía posee una distribución discontinua entre el sector norte de la comuna de Lonquimay y las comunas de Vilcún, Cunco, Melipeuco y Curarrehue por Los Andes, además de una participación baja en la costa (i.e., comunas de Nueva Imperial y Carahue; Fig. 1). La comuna de Lonquimay es la que presenta mayor cobertura de esta especie en La Araucanía y en Chile, con alrededor de 3.432 ha, lo que corresponde a aproximadamente el $37 \%$ de los bosques con presencia de lleuque del país (CONAF 2015).

Prumnopitys andina está descrita como una especie semitolerante a la sombra (Donoso 1981, Rodríguez 2004), que ocupa usualmente estratos intermedios y codominantes en la estructura vertical del bosque, y que de manera excepcional forma rodales puros, participando frecuentemente en bosques con doseles muy abiertos y/o con árboles aislados asociados a cursos de agua (Rodríguez 2004, Amigo et al. 2010, Hechenleitner et al. 2012). Su regeneración natural ocurre mediante semillas, las que se dispersan principalmente por gravedad (Hechenleitner et al. 2012). A pesar de ser una especie escasa, su madera, de albura y duramen blanquecino al ser moderadamente dura (peso específico $\sim 0,48 \mathrm{~g} / \mathrm{cm}^{3}$; Rodríguez 2004) ha sido utilizada para la construcción, producción de rodrigones, fabricación de muebles (Rodríguez 2004) y otros utensilios tradicionales como los ngürewe usados para apretar tejidos de lana a telar (Obs pers).

El lleuque se ha clasificado como especie con problemas de conservación, inicialmente como "rara" (Benoit 1989). Clasificaciones posteriores, utilizando la metodología de la Unión Internacional para la Conservación de la Naturaleza (UICN) indican que lleuque se encuentra en categoría "Vulnerable" (Hechenleitner et al. 2012), calificación que mantiene actualmente el Ministerio del Medio Ambiente (MMA 2012). Se fundamenta la clasificación de "Vulnerable" de esta especie, dada una reducción del tamaño de la población mayor al $30 \%$ en los últimos 30 años, así como una reducción en la calidad del hábitat debido a la deforestación y cambio de uso del suelo hacia plantaciones forestales, principalmente en las regiones del Maule y del Biobío (MMA 2012). En la zona de los andes, se habría constatado una reducción de la distribución de la especie dada la inundación 
de terrenos en la zona de alto Biobío, a fines de los años 90 (Hechenleitner et al. 2012). Como especie "Vulnerable" se establece prohibición de la corta, eliminación, destrucción o descepado, como así mismo la alteración de su hábitat (artículo 19, ley 20283). Cualquier acción que involucre a lleuque debe considerar un plan de Manejo de Preservación y un informe de expertos que debe ser ratificado por CONAF para asegurar que las intervenciones no ponen en riesgo la continuidad de la especie (CONAF 2014a). En este contexto, obras asociadas a centrales de pasada habrían impactado alrededor de 235 individuos en la zona de Melipeuco, donde se establecieron medidas de compensación y restauración asociadas a un área de alrededor de 4 ha (CONAF 2014a). Sin embargo, actualmente no se aplica restricción alguna sobre actividades de recolección de los arilos carnosos de lleuque con objetivos de autoconsumo y/o comercio.

Pese a tratarse de un árbol con problemas de conservación y características singulares dado a su aprovechamiento no maderero, hasta ahora no existen trabajos que se refieran específicamente a la estructura y regeneración de bosques de lleuque. Tampoco es fácil obtener antecedentes relacionados a la producción de arilos carnosos (Rodríguez 2004), pese a que localmente estos son un recurso relevante para el consumo doméstico y venta de productos derivados, como mermeladas y conservas (Hechenleitner et al. 2012). Pese a este uso, también se desconocen aspectos asociados a la producción o extracción de conos de lleuque. En este contexto se hace difícil planear actividades de conservación y/o restauración, al no existir información sobre la dinámica de regeneración de esta especie, la cual es difícil de propagar por semilla dado a que presenta latencia (exógena y endógena) que retrasa su germinación en invernadero por más de un año (Rodríguez 2004)

El objetivo del presente trabajo es caracterizar la estructura y regeneración arbórea en bosques de lleuque al norte de la comuna de Lonquimay ( $38^{\circ} \mathrm{S} 71^{\circ} \mathrm{O}$; Fig. 1). Además de analizar la estructura, (a) se evalúa la regeneración arbórea (diámetro $<5 \mathrm{~cm}$ a $1,3 \mathrm{~m}$ ) y (b) se caracteriza la riqueza y diversidad florística asociada a sitios dominados por lleuque. Se presenta esta información con la idea de indagar a partir de datos empíricos, posibles problemas de regeneración que pudiera tener esta especie.

\section{MÉTODOS}

\section{ÁREA DE ESTUDIO}

El área de estudio se emplaza en el norte de la comuna de Lonquimay, provincia de Malleco, en La Araucanía, específicamente en los sectores de Contraco, Llanquén,
Trobún, Troyo y Paso Paz (38¹'S, 71.30 - 38³'S, 71³' O; Fig. 1). El clima en el área de estudio corresponde al tipo templado cálido con menos de 4 meses secos (Cfsb1; sensu KöppenGeiger), el cual se caracteriza por ser lluvioso, presentando superávit de lluvias entre abril y agosto (Ahumada 1999). La temperatura media anual es de $9,7^{\circ} \mathrm{C}$ y la precipitación media de $1.674 \mathrm{~mm} /$ año, siendo muy comunes las temperaturas bajo cero y precipitaciones de nieve, que pueden alcanzar hasta $2 \mathrm{~m}$ en invierno (Vargas \& Bustamante 2007). Los suelos de esta zona corresponden a andisoles derivados de cenizas volcánicas jóvenes, denominados trumaos. Dada las características orográficas pronunciadas y la fragilidad de estos suelos, más del $60 \%$ de la comuna de Lonquimay presenta algún grado de erosión (Flores et al. 2010).

\section{SELECCIÓN DE BOSQUes PARA MUESTREO}

A partir de la información cartográfica del catastro de recursos vegetacionales nativos de Chile, se consideraron polígonos con presencia de lleuque en el área de estudio, que incluían lleuque como especie categorizada en segunda y tercera dominancia (CONAF 2014b). La dominancia en el catastro está definida como la importancia decreciente de las especies según su estrato y cobertura de copas (CONAF 2017). Al ser lleuque una especie poco abundante, no existen polígonos donde ésta figure en primera dominancia (CONAF 2014b, Donoso 1993, Amigo et al. 2010), Posteriormente, se visitaron los sitios pre-seleccionados para validar la información del catastro. De esta forma, se identificaron áreas para muestreo donde: Ileuque conformaba un bosque $(>0,5$ ha) siendo la especie predominante en términos de árboles por hectárea, el sitio era accesible para muestreo, y existía el conocimiento de habitantes del sector de usar el bosque como zona de recolección tradicional de arilos. Se definieron seis rodales como sitios de muestreo diferenciando la textura en imágenes satelitales de libre disposición utilizando Google Earth (Gorelick et al. 2017). Lleuque pudo ser identificado por tener una copa globosa y mostrar un color verde opaco y asociarse a cursos de agua, correspondiendo principalmente a bosques secundarios adultos ubicados entre los 480 y 950 m.s.n.m (Amigo et al. 2010) (Fig. 1).

\section{Muestreo}

Se establecieron 24 unidades de muestreo de $500 \mathrm{~m}^{2}(20 \mathrm{x}$ $25 \mathrm{~m}$ ) de manera aleatoria en los rodales seleccionados (Fig. 1). En cada unidad de muestreo se registraron variables de sitio como altitud, pendiente y exposición predominante. A todos los árboles con un diámetro a la altura del pecho (1.3 m, DAP) mayor o igual a $5 \mathrm{~cm}$, se les registro la especie, DAP, y la clase de copas (sensu Kraft; Donoso 1993). A una submuestra de árboles, se les midió la altura total ( $h$ ) usando 
un Vertex III, instrumento que incluye un hipsómetro láser (Haglöf $\left.{ }^{\circledR}\right)$. En dos a tres individuos de lleuque dominantes (i.e., del estrato superior y de mayor diámetro) en cada unidad de muestreo, se extrajo un tarugo de incremento en la base del árbol para estimar su edad $(\mathrm{n}=55)$. Así también en cada unidad de muestreo, se establecieron aleatoriamente cuatro sub-parcelas de $4 \mathrm{~m}^{2}$, donde se midió la regeneración arbórea (DAP $<5 \mathrm{~cm}$ ) por clase de altura $(<0,5 \mathrm{~m}, 0,5-2 \mathrm{~m}$ $y>2 m)$, registrando además todas las especies vasculares a través de la escala Braun Blanquet (1979), para estimar la riqueza y diversidad florística. Los taxa vasculares fueron identificados a nivel de especie y clasificados según su origen (nativo/exótico) siguiendo la nomenclatura de Rodriguez et al. (2018). Así también, se contabilizó el número de arilos de lleuque caídos en cada sub-parcela, dado que los muestreos se realizaron en plena época de maduración de la especie (i.e., marzo- abril, 2018; Rodríguez 2004). Finalmente, en cada unidad de muestreo se registraron datos asociados al uso antrópico del bosque, considerando: número de tocones sin rebrotes, como indicador de tala selectiva, y número de bostas, como indicador de densidad de ganado, pisoteo y eventual ramoneo (Zamorano-Elgueta et al. 2014).

\section{ANÁLISIS DE DATOS}

Se calcularon variables de estado de rodal para cada unidad de muestreo, como la densidad ( $N)$, el área basal $(G)$, el diámetro medio del árbol de área basal media $\left(d_{g}\right)$, y la altura dominante $\left(H_{d}\right)$. Esta última variable se estimó mediante el algoritmo propuesto por García (1998). La edad de los individuos dominantes de lleuque se obtuvo en base al conteo y medición de tarugos con lupa, tras ser montados y pulidos (GrissinoMeyer 2003, Liu 1986). En caso de tarugos incompletos (i.e., que no llegaron al centro), se procedió según el algoritmo de Liu (1986). La regeneración arbórea y el número de conos

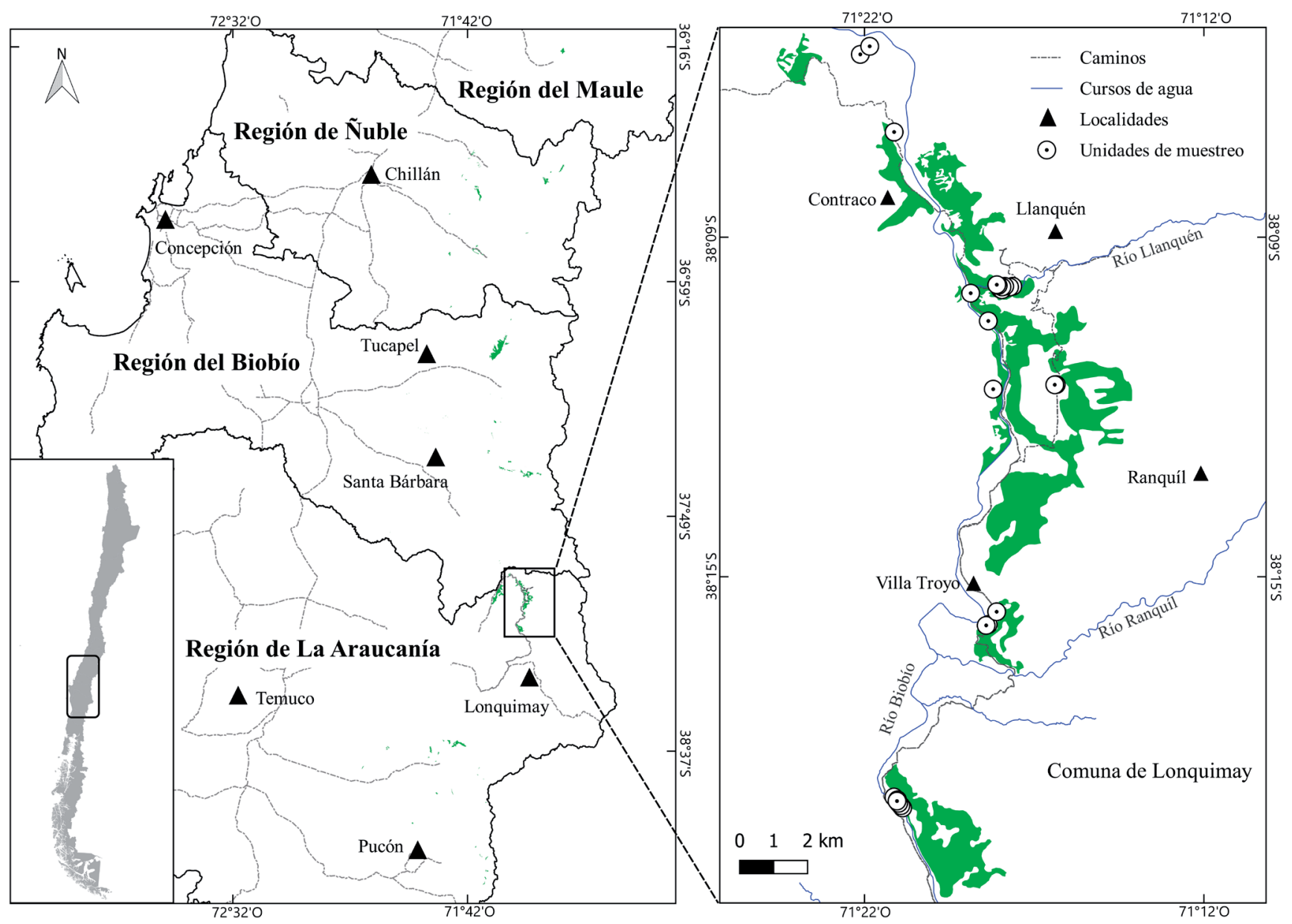

Figura 1. Distribución de P. andina en Chile (en verde sensu CONAF 2016, 2015, 2014a). Derecha: área de estudio en el sector norte de Lonquimay (CONAF 2014a). / Distribution of P. Andina in Chile, (sensu CONAF 2016, 2015, 2014a). On the right is shown the study area in Lonquimay (CONAF 2014a). 
caídos en cada unidad de muestreo, se obtuvo al promediar las respectivas subpacelas. Como indicador de la diversidad de especies vasculares, se estimó la riqueza de especies nativas y exóticas, además del índice de equidad de Pielou, el cual evalúa la similitud de la abundancia relativa de las especies y varía de 0 (dominio de una sola especie) a 1 (abundancia igual de todas las especies, diversidad máxima) (Stirling \& Wilsey 2001). Basado en la marcada diferencia en estructura diamétrica y composición de los bosques muestreados, se presentan los resultados para las siguientes tres condiciones: bosques abiertos, bosques secundarios adultos y bosques puros (i.e. lleuquerías). Esta propuesta se basa en las variables de estado de rodal calculada para cada unidad de muestreo (densidad, área basal y altura dominante, ver Apéndice 1), además se considera la estructura diamétrica de especies y las edades máximas para lleuque. Todos los análisis de datos se realizaron utilizando R (R Development Core Team 2018).

\section{RESULTADOS}

En total, se midieron 1294 individuos arbóreos (> 5 cm DAP), de los cuales $\sim 49 \%$ correspondió a lleuque. Las principales especies acompañantes fueron: N. obliqua (roble; $34 \%$ ), L. hirsuta (radal; 13\%) y A. chilensis (ciprés de la cordillera; 2\%). Otras especies arbóreas menos relevantes en términos de árboles por ha fueron: N. dombeyi, Maytenus boaria Molina y Aristotelia chilensis (Molina) Stunz. Las edades dominantes medianas fluctuaron entre los 66 y 151 años para lleuque, con diámetros medianos que variaron de 25 a $71 \mathrm{~cm}$, y alturas dominantes de $\sim 18 \mathrm{~m}$, destacando individuos de lleuque que presentaron $118 \mathrm{~cm}$ de DAP y hasta $27 \mathrm{~m}$ de altura. En la Tabla 1 se presentan valores medianos de las variables de sitio, variables de estado y variables de riqueza y diversidad.

TABLA 1. Medianas de variables de sitio, de estado de rodal y de riqueza de especies vasculares, según tipo de bosques con presencia de lleuque. El coeficiente de variación porcentual se representa entre paréntesis. Se presentan las principales especies arbóreas encontradas. / Median of site variables, stand variables and vascular plant species richness, according to each forest structure dominated by lleuque. The coefficient of variation is given in parentheses.

\begin{tabular}{|c|c|c|c|}
\hline Variable/Estructura & Bosques abiertos & $\begin{array}{l}\text { Bosques secundarios } \\
\text { adultos }\end{array}$ & $\begin{array}{l}\text { Bosques puros } \\
\text { "lleuquerías" }\end{array}$ \\
\hline \multicolumn{4}{|l|}{ (a) Variables de sitio } \\
\hline Altitud (m.s.n.m) & $757(22)$ & 854 (14) & $947(0,3)$ \\
\hline Pendiente (\%) & $61(52)$ & $51(41)$ & $16(9)$ \\
\hline Exposición predominante & NO & NE & NO \\
\hline \multicolumn{4}{|l|}{ (b) Variables de estado } \\
\hline \multicolumn{4}{|l|}{ Densidad (árb/ha) } \\
\hline Prumnopitys andina & $280(60)$ & $570(64)$ & $600(0)$ \\
\hline Nothofagus obliqua & $300(43)$ & $210(97)$ & 0 \\
\hline Lomatia hirsuta & 40 (128) & $140(69)$ & 0 \\
\hline Austrocedrus chilensis & 0 & 0 (185) & 0 \\
\hline Maytenus boaria & $0(200)$ & $0(424)$ & 0 \\
\hline Aristotelia chilensis & 0 & $0(424)$ & 0 \\
\hline Total & $710(14)$ & $980(29)$ & $600(0)$ \\
\hline $\mathrm{DMC}(\mathrm{cm})$ & $26(29)$ & $25(22)$ & $71(1)$ \\
\hline P. andina & $18(42)$ & $20(62)$ & $71(1)$ \\
\hline Altura dominante (m) & $18,4(25)$ & $18,3(18)$ & $18(14)$ \\
\hline P. andina & $26(0)$ & $13,4(28)$ & $18(14)$ \\
\hline Área basal (m²/ha) & $41,3(44)$ & $43,2(35)$ & $237,1(3)$ \\
\hline P. andina & $9,4(85)$ & $15,6(74)$ & $237,1(3)$ \\
\hline \multicolumn{4}{|l|}{ Edad dominante (años) } \\
\hline P. andina & $65,7(41)$ & $72,6(31)$ & $150.6(31)$ \\
\hline \multicolumn{4}{|c|}{ (c) Riqueza y diversidad florística } \\
\hline Riqueza de especies nativas & $2(40)$ & $3,5(48)$ & $1(37)$ \\
\hline Riqueza de especies exóticas & $3(54)$ & $2(79)$ & $2(57)$ \\
\hline Índice de equidad (Pielou J) & $0,46(68)$ & $0.4948)$ & 0,14 129) \\
\hline
\end{tabular}


La densidad mediana varió entre 600 a 980 árb/ha. Bosques secundarios adultos presentaron densidades mayores, pero áreas basales similares a las de bosques abiertos. Los bosques puros o "lleuquerías" destacaron por el gran diámetro de sus individuos $(>70 \mathrm{~cm})$, que acumulan un área basal extraordinaria $>200 \mathrm{~m}^{2}$ (Tabla 1 ).

Los valores medianos de riqueza de plantas vasculares fluctuaron entre 1 y 3,5 para especies nativas y entre 2 y 3 especies exóticas (Tabla 1), no existiendo diferencias marcadas entre los tipos de estructuras evaluadas. El índice de equidad de Pielou, en tanto, fue particularmente bajo para el caso de bosques puros de lleuque (Tabla 1). Un listado de todas las especies vasculares por tipo de bosques se presenta en el Apéndice 1.
Las distribuciones diamétricas varían considerablemente para los tres tipos de bosques (Fig. 2). La distribución de tamaños en bosques abiertos fue relativamente uniforme, lleuque destaca en este tipo de estructura como especie dominante en las clases diamétricas inferiores y superiores (Fig. 2). Los bosques secundarios adultos presentan gran cantidad de individuos en las clases diamétricas inferiores (< $30 \mathrm{~cm}$ DAP) compuestos principalmente por roble, lleuque y radal (Fig. 2, Tabla 1). Los bosques puros, o lleuquerías presentaron una baja densidad, compuestos exclusivamente por individuos de lleuque de gran tamaño, y ausencia de clases diamétricas $<30 \mathrm{~cm}$ (Fig. 2), con árboles dominantes de más de 150 años (Tabla 1).

TABLA 2. Regeneración mediana de las principales especies arbóreas según clase de altura en bosques de lleuque. El coeficiente de variación porcentual se muestra entre paréntesis. / Median regeneration of the main tree species by height classes. The coefficient of variation is shown in parentheses.

\begin{tabular}{|c|c|c|c|c|c|c|c|c|c|}
\hline \multirow{2}{*}{$\begin{array}{l}\text { Densidad de la } \\
\text { regeneracion }\end{array}$} & \multicolumn{3}{|c|}{ Bosques abiertos } & \multicolumn{3}{|c|}{ Bosques secundarios adultos } & \multicolumn{3}{|c|}{ Bosques puros "Ileuquerías" } \\
\hline & $>0-0,5 \mathrm{~m}$ & $>05-2 m$ & $>2 m$ & $>0-0,5 \mathrm{~m}$ & $>05-2 m$ & $>2 m$ & $>0-0,5 \mathrm{~m}$ & $>05-2 m$ & $>2 \mathrm{~m}$ \\
\hline Aristotelia chilensis & 625 & 2500 & 1875 & $1875(117)$ & $0(237)$ & & & & \\
\hline Austrocedrus chilensis & & & & 1250 & & & & & \\
\hline Lomatia hirsuta & $6562(127)$ & 0 & $2500(141)$ & $1250(147)$ & $0(269)$ & $0(198)$ & & & \\
\hline Maytenus boaria & & & & $625(0)$ & & & & & \\
\hline Maytenus disticha & & & & $0(185)$ & & & & & \\
\hline Nothofagus dombeyi & & & & $1562(103)$ & & & & & \\
\hline Nothofagus obliqua & $1562(141)$ & 0 & $1875(141)$ & $1562(203)$ & $0(195)$ & & & & \\
\hline Prumnopitys andina & $3125(141)$ & 0 & $625(0)$ & $3750(94)$ & $0(178)$ & $0(201)$ & $25937(28)$ & & \\
\hline Otras & $1875(47)$ & 0 & 0 & $625(64)$ & 0 & 0 & 625 & 0 & 0 \\
\hline Total & 1250 (136) & $0(300)$ & 325 (141) & $1250(172)$ & $0(323)$ & $0(363)$ & 20625 (88) & 0 & 0 \\
\hline
\end{tabular}

TABLA 3. Variables asociadas a influencia antrópica, y arilos de lleuque encontrados en el suelo de las diferentes estructuras estudiadas. Se presentan medianas y coeficiente de variación (\%). / Variables associated with anthropic influence, and fleshy cones of lleuque found in the soil of the different forest structures dominated by lleuque. Medians and coefficients of variation (\%) is provided.

\begin{tabular}{lccc}
\hline Variable & Bosques abiertos & Bosques secundarios adultos & Bosques puros "lleuquerías" \\
\hline Tocones (nr/ha) & $40(86)$ & $70(109)$ & $100(56)$ \\
Bostas (nr/ha) & $150(206)$ & $0(243)$ & $300(141)$ \\
Arilos caídos (nr/ha) & $0(200)$ & $0(364)$ & $3906(84)$ \\
\hline
\end{tabular}



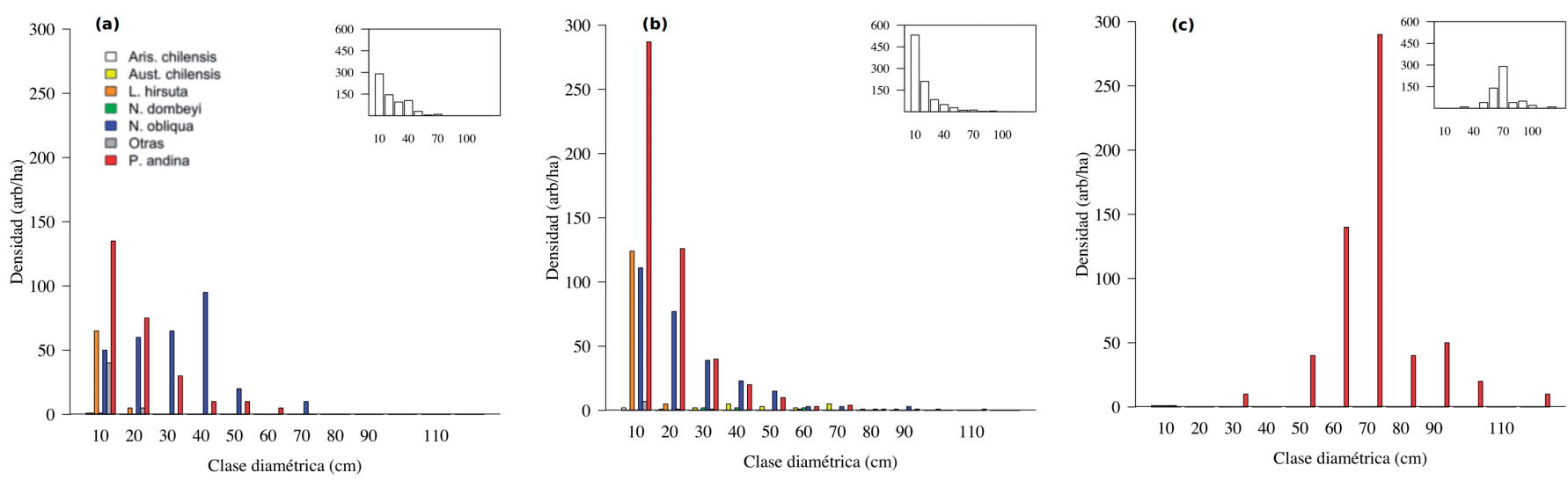

Figura 2. Distribución diamétrica para los tres tipos de estructuras evaluadas: bosques abiertos (a), bosques secundarios adultos (b) y bosques puros o "lleuquerías" (c). Diferentes colores representan distintas especies. En barras blancas al extremo derecho de cada figura, se presenta la distribución diamétrica de todas las especies. / Histograms of the diametric distribution for the forest structures dominated by lleuque, (a) open forests, (b) secundary mature forests and (c) pure forests of lleuque. Different species are shown with different colors. In white bars and at the right upper corner of each figure, the diametric distribution of all species is presented.

Los tres tipos de estructura evaluados presentaron regeneración arbórea (< $5 \mathrm{~cm}$ Dap, Tabla 2). Bosques abiertos y especialmente los bosques secundarios adultos mostraron individuos regenerando en las tres categorías de altura consideradas. Esto no ocurre en las lleuquerías, pese a la muy alta densidad de lleuque que logra germinar, reflejándose en la categoría < 0,5 m de altura que acumula más de 25 mil individuos por hectárea (Tabla 2).

Al considerar las variables asociadas a influencia antrópica, los valores medianos de tocones por ha, fluctuaron entre $40 \mathrm{y}$ 100, existiendo en todos los tipos de estructura presencia de ganado (Tabla 3). Arilos caídos se observaron particularmente en las lleuquerías que acumularon gran cantidad.

\section{DISCUSIÓN}

Lleuque ocurre en el área de estudio formando bosques abiertos, bosques secundarios adultos y menos frecuentemente, bosques puros o lleuquerías. Estas formaciones difieren en su estructura y composición dependiendo de su estado de desarrollo y/o historia de uso. Los bosques abiertos y los bosques secundarios adultos se componen principalmente por roble y lleuque, además de radal y Maytenus boaria (maitén), acumulando entre 700 y 1000 individuos por hectárea, respectivamente. Ambos tipos de estructura no varían demasiado en área basal $\left(\sim 40 \mathrm{~m}^{2} /\right.$ ha), pero si en la proporción de lleuque, que suma más del doble de individuos por hectárea en bosques secundarios adultos, que en bosques abiertos. Los bosques puros o lleuquerías constituyen una condición excepcional y son considerablemente más escasos que los bosques abiertos y bosques secundarios adultos, presentando sólo individuos de esta especie en su estructura arbórea ( 600 arb/ha). Con un área basal extraordinaria, de más de $200 \mathrm{~m}^{2}$, esta gran acumulación de biomasa, sólo se puede comparar a la que presenta Fitzroya cupressoides (Molina) I.M. Johnst en combinación con especies siempreverdes en bosques de la costa y los andes más al sur (Donoso 1993, Lara 1991). De manera similar, otros bosques dominados por podocarpaceas que incluyen especies de Prumnopitys spp. en Nueva Zelanda, acumulan también áreas basales considerables, mayores a 120 $\mathrm{m}^{2} /$ ha (Lusk 2002). Cabe destacar que los diámetros y alturas medidos para lleuque en el presente trabajo, corresponden a los más grandes reportados para la especie, con individuos de más de $23 \mathrm{~m}$ de altura y $118 \mathrm{~cm}$ de diámetro (Hechenleitner et al. 2012, Rodríguez et al. 2005)

En general, los datos evaluados en este trabajo corroboran a lleuque como un árbol escaso, poco frecuente en los bosques donde participa, que raramente domina y/o forma bosques puros (Amigo et al. 2010, CONAF 2017). Sin embargo, una vez que la especie ocurre en un sitio determinado, parece ser abundante, explicándose esto por su copiosa regeneración a partir de semillas. Lleuque está presente en todas las clases diamétricas en los bosques abiertos y particularmente en los bosques secundarios adultos, presentando ciertas 
clases inferiores ausentes ( $<30 \mathrm{~cm}$ DAP) sólo en el caso de los bosques puros. Esto último dado por una mayor presión antrópica en los rodales puros evaluados y no por características auto-ecológicas. La especie no presentaría mayores problemas de regeneración (Tabla 2), siendo aparentemente semitolerante a sombratolerante, dado a que logra regenerar en buen número, en el sotobosque de todas las estructuras estudiadas ( 3000 ind/ha). Esto se condice con los atributos de otras podocarpáceas de los bosques del sur de Chile, como Saxegothaea consipicua y Podocarpus nubigena, las cuales presentan un modo de regeneración continua (Lusk 1996, Christie \& Armesto 2003). Destaca la gran cantidad de individuos ( $<0,5 \mathrm{~m}$ de altura) que germinan en las lleuquerías. Sin embargo, estos no lograrían reclutar a las siguientes categorías de regeneración (>0,5 m), lo cual se explica dada la mayor presión antrópica evidenciada en este tipo de estructura, que presentó mayor número de tocones y de bostas (Tabla 3; Zamorano-Elgueta et al., 2014). Es plausible que los bosques puros de lleuque sean reflejo de un estado sucesional avanzado, acelerado por presión antrópica, dada la tala selectiva de especies acompañantes de interés maderero y leñero, como roble y radal, respectivamente. De esta manera, los bosques secundarios adultos, en ausencia de disturbios de envergadura, podrían fácilmente convertirse en lleuquerías. En concordancia con los resultados de este trabajo, Hechenleitner et al. (2012) mencionan que, en sitios parcialmente deforestados para uso ganadero, se suelen mantener en pie los lleuques adultos (probablemente dado el uso no maderero de sus arilos carnosos, Obs pers). La especie finalmente no reclutaría también, dado que cerdos y cabras se alimentan de sus semillas carnosas (Hechenleitner et al. 2012, Amigo et al., 2010). Esta mayor presión antrópica por ganado en las lleuquerías, determinaría también diferencias florísticas que explican la homogeneidad encontrada en los bosques puros que son menos diversos en términos de frecuencia y abundancia de plantas que bosques abiertos $y$ bosques secundarios adultos (Apéndice 2).

\section{CONCLUSIONES}

Este trabajo reporta por primera vez para la ciencia, aspectos estructurales de bosques dominados por la especie vulnerable: Iluque, Prumnopytis andina ocurre en tres tipos de estructura: bosques abiertos, bosques secundarios adultos, y bosques puros o "lleuquerías". La densidad de lleuque varía entre 280 a 600 árboles por ha, con un área basal mediana de entre 41 y $237 \mathrm{~m}^{2} / \mathrm{ha}$. La edad máxima estimada para los ejemplares dominantes de lleuque varió entre 66 y 151 años, presentando siempre abundante regeneración (entre 3 mil y 25 mil ind/ ha). La riqueza total en bosques dominados por lleuque fue de 52 especies vasculares (29 nativas, 23 exóticas), siendo algo más diversos los bosques secundarios adultos que los bosques abiertos y bosques puros. En ausencia de disturbios antrópicos lleuque no presentaría problemas de regeneración que pudieran afectar su conservación. Sin embargo, el ramoneo por ganado, sumado al sobrepastoreo y eventual consumo de conos por animales, parecen estar determinando la ausencia de reclutamiento observada, particularmente en bosques puros.

\section{AGRADECIMIENTOS}

Agradecemos a los proyectos: FIA: PYT-2017-0694 , FIBN No. 048/2019 y FONDECYT iniciación 11170987. Se agradece la ayuda en terreno y laboratorio de: Nicole Salas, Deyanira Cortés, Carla Manríquez, Constanza Garcés, Mabel Pavéz, Paola Arroyo y Nayadeth Muñoz del Laboratorio de Biometría de la Universidad de La Frontera. Finalmente, se agradece a los propietarios del sector norte de la comuna de Lonquimay, en especial a Gloria Palma (Llanquén), Jenny Sobarso (Paso Paz) y a la Sra. Ana Fuentealba, por su hospitalidad en Villa Troyo.

\section{REFERENCIAS}

Amigo, J., Rodríguez-Guitián, M.A., Ramírez, C. 2010. The Lleuque forests of South-Central Chile: a phytosociological study and syntaxonomical classification within South American temperate forests. Lazaroa 31: 85-98.

Ahumada, C., Palma, T., Centrón, L. 1999. Pauta de condición de las veranadas en la IX Región de La Araucanía.Ministerio de Agricultura (SAG). Departamento de Protección de los Recursos Naturales Renovables 9: 1-71.

CONAF. 2014a. Resolución No: 514/2014 Ant: Solicitud de excepcionalidad del Artículo 19 de la Ley $N^{\circ} 20.283$. Corporación Nacional Forestal, Santiago, Chile. 13 pp.

CONAF. 2014b. Monitoreo de cambios, corrección cartográfica y actualización del Catastro de los recursos vegetacionales nativos de la Región de La Araucanía. Universidad Austral, Universidad de La Frontera, Corporación Nacional Forestal, Santiago, Chile. 42 pp.

CONAF. 2017. Monitoreo de cambios, corrección cartográfica y actualización del catastro de los recursos vegetacionales nativos de la Región del Biobío. Departamento de Monitoreo de Ecosistemas Forestales, Corporación Nacional Forestal, Santiago, Chile. 74 pp.

Donoso, C. 1981. Tipos forestales de los bosques nativos de 
Chile. Documento de trabajo 38.70 pp.

Donoso, C. 1993. Bosques templados de Chile y Argentina. Variación, estructura y dinámica. Editorial Universitaria, Santiago, Chile. 483 pp.

Flores, J.P., Torres, C., Martínez, E., Muñoz, P. 2010. Determinación de la erosión actual y potencial de los suelos en la Isla de Pascua. Informe final Pub. CIREN N 177/2013. 82 pp.

García, O. 1998. Estimating top height with variable plot sizes. Canadian Journal of Forest Research 28(10): 1509-1517.

Gorelick, N., Hancher, M., Dixon, M., Ilyushchenko, S., Thau, D. Moore, R. 2017. Google Earth Engine: Planetary-scale geospatial analysis for everyone. Remote Sensing of Environment 202: 18-27.

Grissino-Mayer, H.D. 2003. A Manual and Tutorial for the Proper Use of an Increment Borer. Tree-Ring Research 59(2): 63-79.

Hechenleitner, P., Gardner, M., Thomas, P., Echeverrı, C., Escobar, B., Brownless, P., Martinez, C. 2012. Plantas Amenazadas del Centro-sur de Chile. Segunda edición. Universidad Austral de Chile, Real Jardín Botánico de Edimburgo. Marisa Cuneo Ediciones.

Lara, A. 1991. The Dynamics and Disturbance Regimes of "Fitzroya cupressoides" Forests in the South-Central Andes of Chile. PhD Tesis. University of Colorado, Boulder. 183 pp.

Liu, C.J. 1986. Rectifying radii on off-center increment cores. Forest Science 32(4): 1058-1061.

Lusk, C.H. 1996. Stand dynamics of the shade-tolerant conifers Podocarpus nubigena and Saxegothaea conspicua in Chilean temperate rain forest. Journal of Vegetation Science 7(4): 549-558.
Lusk, C.H. 2002. Basal area in a New Zealand podocarpbroadleaved forest: Are coniferous and angiosperm components independent? New Zealand Journal of Botany 40: 143-147.

MMA. 2012. Ficha de antecedentes de especies, Prumnopitys andina (Poepp. ex Endl.) de Laub. Ministerio del Medio Ambiente, Chile. http://www.mma.gob.cl/ clasificacionespecies/fichas9proceso/FICHAS_ INICIO_9o_PROCESO_PDF/Prumnopitys_andina.pdf

R Development Core Team. 2018. R: A language and environment for statistical computing. R version 3.2.3 The R Foundation for Statistical Computing, Vienna, Austria. URL: https:// www.R-project.org/.

Rodriguez, R. 2004. Lleuque (Prumnopitys andina): Especie con problemas de conservación en Chiloé. ENDESA, Santiago, Chile. 47pp.

Rodriguez, R.R., Matthei, S.O., Quezada, M.M. 1983. Flora arbórea de Chile. Chile, Editorial de La Universidad de Concepción. 408 pp.

Rodriguez, R., Marticorena, C., Alarcón, D., Baeza, C., Cavieres, L., Finot, V.L., Fuentes, N., Klessling, A., Mihoc, M., Pauchard, A., Ruiz, E., Sanchez, P., Marticorena, A. 2018. Catálogo de las plantas vasculares de Chile. Gayana Botánica 75(1): $1-430$

Vargas-Gaete, R., Bustamante, P. 2007. Silvicultura social: un estudio de caso en el sector Llanquén, Lonquimay. Revista Bosque Nativo 41(1): 3-9.

Zamorano-Elgueta, C., Cayuela, L., Rey-Benayas, J.M., Donoso, P.J., Geneletti, D., Hobbs, R.J. 2014. The differential influences of human-induced disturbances on tree regeneration community: a landscape approach. Ecosphere 5(7): 1-17.

APENDICE 1. Variables de estructura de rodal para cada una de las unidades de muestreo. / Stand structure variables for each of the sample units.

\begin{tabular}{ccccccc}
\hline $\begin{array}{c}\text { No. } \\
\text { Parcela }\end{array}$ & $\begin{array}{c}\text { Densidad } \\
(\mathrm{arb} / \mathrm{ha})\end{array}$ & $\begin{array}{c}\text { Área basal } \\
\left(\mathrm{m}^{2} / \mathrm{ha}\right)\end{array}$ & $\begin{array}{c}\text { Altura } \\
\text { dominante } \\
(\mathrm{m})\end{array}$ & $\begin{array}{c}\text { Densidad } \\
\text { lleuque } \\
(\mathrm{arb} / \mathrm{ha})\end{array}$ & $\begin{array}{c}\text { Área basal } \\
\text { leuque } \\
\left(\mathbf{m}^{2} / \mathrm{ha}\right)\end{array}$ & Estructura \\
\hline 1 & 680 & 16,3 & 11,3 & 440 & 8,6 & Bosque abierto \\
2 & 540 & 54,2 & 21,2 & 240 & 23,5 & Bosque abierto \\
3 & 760 & 48,7 & 17,0 & 320 & 10,2 & Bosque abierto \\
4 & 740 & 33,9 & 19,8 & 60 & 1,2 & Bosque secundario adulto \\
5 & 920 & 40,5 & 13,6 & 620 & 20,3 & Bosque secundario adulto \\
6 & 800 & 43,8 & 19,9 & 200 & 1,8 & Bosque secundario adulto \\
7 & 780 & 20,7 & 18,5 & 620 & 6,6 & Bosque secundario adulto \\
8 & 700 & 40,5 & 19,0 & 480 & 13,3 & Bosque secundario adulto \\
9 & 1180 & 37,1 & 18,2 & 340 & 6,7 & Bosque secundario adulto \\
\hline
\end{tabular}




\begin{tabular}{ccccccc}
\hline $\begin{array}{c}\text { No. } \\
\text { Parcela }\end{array}$ & $\begin{array}{c}\text { Densidad } \\
(\mathrm{arb} / \mathrm{ha})\end{array}$ & $\begin{array}{c}\text { Área basal } \\
\left(\mathrm{m}^{2} / \mathrm{ha}\right)\end{array}$ & $\begin{array}{c}\text { Altura } \\
\text { dominante } \\
(\mathrm{m})\end{array}$ & $\begin{array}{c}\text { Densidad } \\
\text { lleuque } \\
(\mathrm{arb} / \mathrm{ha})\end{array}$ & $\begin{array}{c}\text { Área basal } \\
\text { lleuque } \\
\left(\mathrm{m}^{2} / \mathrm{ha}\right)\end{array}$ & Estructura \\
\hline 10 & 1080 & 38,0 & 13,8 & 840 & 15,2 & Bosque secundario adulto \\
11 & 1080 & 72,3 & 19,3 & 880 & 32,4 & Bosque secundario adulto \\
12 & 1200 & 40,3 & 20,6 & 840 & 15,4 & Bosque secundario adulto \\
13 & 820 & 61,5 & 16,6 & 420 & 15,8 & Bosque secundario adulto \\
14 & 720 & 44,0 & 25,9 & 20 & 7,5 & Bosque secundario adulto \\
15 & 1020 & 59,4 & 25,6 & 260 & 47,9 & Bosque secundario adulto \\
16 & 840 & 83,0 & 19,2 & 280 & 42,0 & Bosque secundario adulto \\
17 & 800 & 49,5 & 15,2 & 580 & 46,9 & Bosque secundario adulto \\
18 & 1860 & 65,4 & 16,7 & 1520 & 30,1 & Bosque secundario adulto \\
19 & 940 & 51,3 & 18,2 & 600 & 28,6 & Bosque secundario adulto \\
20 & 1520 & 28,3 & 19,9 & 80 & 2,8 & Bosque secundario adulto \\
21 & 1240 & 25,0 & 16,9 & 560 & 8,7 & Bosque secundario adulto \\
22 & 1220 & 42,6 & 16,8 & 720 & 16,3 & Bosque secundario adulto \\
23 & 600 & 241,6 & 16,3 & 600 & 241,6 & Bosque puro \\
24 & 600 & 232,7 & 19,9 & 600 & 232,7 & Bosque puro \\
\hline
\end{tabular}

APENDICE 2. Listado de plantas vasculares asociadas a los diferentes bosques de lleuque estudiados. / List of vascular plants associated with the different forest structures dominated by lleuque.

\begin{tabular}{|c|c|c|c|c|}
\hline Especie & $\begin{array}{l}\text { Bosques } \\
\text { abiertos }\end{array}$ & $\begin{array}{c}\text { Bosques } \\
\text { secundarios adultos }\end{array}$ & Bosques puros & Origen \\
\hline Acaena ovalifolia (Ruiz \& Pav) & & $x$ & & Nativa \\
\hline Agrostis spp. & & $x$ & & Exótica \\
\hline Alstroemeria spp. & & $x$ & & Nativa \\
\hline Aristotelia chilensis (Molina) Stuntz & $x$ & $x$ & & Nativa \\
\hline Austrocedrus chilensis (D. Don) Pic. Serm. \& Bizzarri & & $\mathrm{x}$ & & Nativa \\
\hline Azara microphylla (Hook.f) & $x$ & $x$ & & Nativa \\
\hline Berberis darwinii (Hook. F) & $x$ & $x$ & & Nativa \\
\hline Berberis microphylla (G. Forst) & & $x$ & & Nativa \\
\hline Blechnum hastatum (Kaulf.) & & $x$ & & Nativa \\
\hline Blechnum microphyllum (Goldm.) C.V. Morton & & $x$ & & Nativa \\
\hline Chusquea spp. & $x$ & $x$ & & Nativa \\
\hline Colletia spinosissima (J.F.Gmel.) & $x$ & & & Nativa \\
\hline Conium maculatum (L.) & & $x$ & & Exótica \\
\hline Crepis capillaris (L.) & $x$ & $x$ & & Exótica \\
\hline Cyperaceae spp. & $x$ & & & Nativa \\
\hline Echium vulgare (L.) & & $\mathrm{x}$ & & Exótica \\
\hline
\end{tabular}




\begin{tabular}{|c|c|c|c|c|}
\hline Especie & $\begin{array}{l}\text { Bosques } \\
\text { abiertos }\end{array}$ & $\begin{array}{c}\text { Bosques } \\
\text { secundarios adultos }\end{array}$ & Bosques puros & Origen \\
\hline Embothrium coccineum J.R. Forst. \& G. Forst & & $x$ & & Nativa \\
\hline Equisetum bogotense Kunth & & $x$ & & Exótica \\
\hline Fragaria chiloensis (L.) Mill & $x$ & $x$ & & Nativa \\
\hline Galium spp. & & $x$ & & Nativa \\
\hline Gaultheria mucronata (L.f.) Hook. \& Arn. & & $x$ & & Nativa \\
\hline Gaultheria phillyreifolia (Pers.) Sleumer & & $x$ & & Nativa \\
\hline Geranium core-core (Steud.) & $x$ & $x$ & $x$ & Nativa \\
\hline Holcus lanatus (L.) & $x$ & & & Exótica \\
\hline Hypericum perforatum L. & & $x$ & & Exótica \\
\hline Hypochaeris radicata (L.) & $x$ & $x$ & $x$ & Exótica \\
\hline Lactuca serriola (L.) & $x$ & & & Exótica \\
\hline Leucanthemum vulgare (Lam.) & & $x$ & & Exótica \\
\hline Lomatia hirsuta (Lam.) Diels & $x$ & $x$ & & Nativa \\
\hline Lotus spp. & & $x$ & & Exótica \\
\hline Maytenus boaria (Molina) & & $x$ & & Nativa \\
\hline Maytenus disticha (Hook.f.) Urb & & $x$ & & Nativa \\
\hline Mentha pulegium (L.) & & $x$ & & Exótica \\
\hline Myoschilos oblongum (Ruiz \& Pav) & $x$ & $x$ & & Nativa \\
\hline Nothofagus dombeyi (Mirb.) Oerst. & & $x$ & & Nativa \\
\hline Nothofagus obliqua (Mirb.) Oerst & $x$ & $x$ & & Nativa \\
\hline Osmorhiza chilensis (Hook. \& Arn.) & & $x$ & $x$ & Nativa \\
\hline Plantago major (L.) & $x$ & & & Exótica \\
\hline Poa spp. & $x$ & $x$ & $x$ & Exótica \\
\hline Prumnopitys andina (Poepp. ex Endl.) de Laub & $x$ & $x$ & $x$ & Nativa \\
\hline Prunella vulgaris (L.) & $x$ & $x$ & $x$ & Exótica \\
\hline Prunus spp. & $x$ & $x$ & $\mathrm{x}$ & Exótica \\
\hline Pyrus communis (L.) & $x$ & & & Exótica \\
\hline Ribes spp. & & $x$ & & Nativa \\
\hline Rosa eglanteria (L.) & $x$ & $x$ & & Exótica \\
\hline Rumex acetosella (L.) & $x$ & $x$ & $x$ & Exótica \\
\hline Schinus polygamus (cav.) cabrera & $x$ & & $x$ & Nativa \\
\hline Senecio vulgaris (L.) & $x$ & $x$ & & Exótica \\
\hline Solanum valdiviense Dunal & & $x$ & & Nativa \\
\hline Trifolium repens (L.) & $x$ & & $x$ & Exótica \\
\hline Tussilago farfara (L.) & & $x$ & & Exótica \\
\hline Verbascum thapsus (L.) & & $x$ & & Exótica \\
\hline
\end{tabular}

Received: 18.11.2019

Accepted: 19.04.2020 